\title{
Das Subjekt des Lernens
}

\author{
Elke Gruber
}

Online publiziert: 5. März 2015

(C) Die Autor(en) 2015. Dieser Artikel ist auf Springerlink.com mit Open Access verfügbar.

\section{Rezension zu:}

Peter Schlögl (2014). Ästhetik der Unabgeschlossenheit. Das Subjekt des lebenslangen Lernens. Transcript Verlag, Bielefeld, 333 Seiten, 29,99€, ISBN978-3-531-8376-2643-8

Wer kennt sie nicht, die Polemiken zum lebenslangen Lernen? Sie sind (fast) so alt, wie der Begriff selbst, der in seiner aktuellen Ausprägung als politisches Instrument der Bewältigung sozialer Transformationsprozesse seinen Siegeszug in den 1990er Jahren angetreten hat. In diesem Kontext ist von lebenslänglicher Erziehung die Rede, vom Missbrauch des Konzepts als neue Regierungspraxis im Rahmen der Konstituierung des unternehmerischen Selbst oder gar von einer totalen Pädagogisierung. Kritik ist durchaus angebracht - zumal die normative Auslegung und Verwendung der Idee des lebenslangen Lernens sowohl in der bildungspolitischen Programmatik als auch in der pädagogischen Praxis überwiegt. Doch sollte man aufgrund dieser Interpretationen das Konzept als solches ,"̈̈ber Bord werfen“? Nein - ist die klare Antwort des Autors des vorliegenden Buches. Vielmehr geht es darum, das Subjekt stärker als bisher zum Dreh- und Angelpunkt der Bemühungen um lebenslange Lernprozesse zu machen. Nun ist das zwar nicht neu, so deutlich wurde es jedoch lange nicht gesagt und vor allem theoriegeleitet begründet.

Im Unterschied zum üblichen psychologischen oder bildungspolitischen Zugang zum (lebenslangen) Lernen wählt der Autor einen philosophischen Zugang, der Lernen in den Kontext allgemein-menschlicher Entwicklungs- und Entfaltungsprozesse stellt. Im Zentrum seiner Argumentation, der aufgrund der Fülle an unterschiedlichen Zugängen, Strängen und Verweisen manchmal etwas schwer zu folgen ist, steht die Unabgeschlossenheit von Lernprozessen. Diese wird im Hinblick auf zwei Dimensionen diskutiert: Zum einen auf die nicht abschließbare Phase von Bildung im

Univ.-Prof. Dr. E. Gruber $(\bowtie)$

Institut für Erziehungswissenschaft und Bildungsforschung (IFEB),

Graz, Österreich

E-Mail: elke.gruber@uni-graz.at 
gesamten Lebenslauf, zum anderen auf die Entgrenzung des Lernens über klassische Vorstellungen von Bildung hinaus. Um letzteres, nämlich die ,Würdigung lebenspraktischer Erfahrungen“ (S. 163), kreist dann auch die theoretische Auseinandersetzung des Autors. Zuvor findet sich ein lesenswerter Abriss über die Genese und Erscheinungsformen des Diskurses zum lebenslangen Lernen, der nicht nur einige interessante Details über die Vorläufer der Programmatik enthalt (wie den Bericht des Erwachsenenbildungskomitees des britischen Ministeriums für Wiederaufbau aus dem Jahre 1919), sondern auch begriffsetymologische Präzisierungen vornimmt.

In seiner Hauptargumentation greift der Autor dann auf zwei im deutschsprachigen Raum bisher im Kontext des lebenslangen Lernens wenig rezipierte Theoriestränge zurück: die geisteswissenschaftliche Pädagogik wie sie sich in den Werken der sogenannten Klassiker der deutschen Berufspädagogik wie Georg Kerschensteiner, Eduard Spranger, Theodor Litt u. a., widerspiegelt und der auf dem amerikanischen Transzendentalismus sowie dem daraus erwachsenden Pragmatismus beruhenden Pädagogik John Deweys. Zentrales Element - bei aller Unterschiedlichkeit der Ansätze - ist die Tatsache, dass Bildung und Entwicklung nicht das Resultat der Aneignung eines wie immer gearteten, mehr oder weniger geschlossenen Bildungskanons ist, sondern in der tätigen Auseinandersetzung mit sich, mit anderen und mit der Umwelt erfolgt. Erfahrung spielt hier eine zentrale Rolle, sie ist nicht nur der Ausgangspunkt jeglicher Bildungsprozesse, sondern sie lässt den Menschen auch teilhaben am gesellschaftlichen Gemeinwesen, was wiederum eine notwendige Voraussetzung für Demokratisierungsprozesse ist. Erfahrung in diesem Sinne gedacht geht über die klassische pädagogische Kategorie einer „Mittel-zum-Zweck-Relation“ hinaus und wird zum universellen Element menschlicher Entwicklung und somit des Lebens. So gesehen sind die Schlussfolgerungen, die der Autor für das (lebenslange) Lernen zieht, folgerichtig: Es geht nicht darum, Bildung als ein abschließbares „Ganzes“ zu sehen, sondern Lernen im Sinne eines „existentielle(n) Verhältniss(es) zur Welt“ (S. 163) ist ein offenes Projekt, das „Learning to know, Learning to do, Learning to live together, Learning to live with others sowie Learning to be“ (S. 200) umfasst. Wie dies dann tatsächlich umgesetzt werden kann, welche pädagogischen Konzepte dafür zielführend sind und welcher Rahmenbedingungen es bedarf, wäre einen Folgeband wert.

Im Ganzen liefert die Publikation einen originären Beitrag zur Diskussion um eine theoriegeleitete Fundierung des Konzepts des lebenslangen Lernens. Darüber hinaus gibt sie Einblick in manch interessante Details zu den historischen Quellen und der Begriffsgeschichte des lebenslangen Lernens. Die manchmal etwas „umwegige“ Argumentation und der vor allem im Einleitungskapitel etwas schwer verständliche Sprachstil sind wohl der Tatsache geschuldet, dass es sich beim vorliegenden Buch um eine Graduierungsarbeit handelt. Die den einzelnen Kapiteln vorangestellten Zusammenfassungen machen diese kleine Schwäche der Publikation aber wieder wett. Das Buch sei all jenen Leserinnen und Lesern empfohlen, die sich mit Grundfragen der Erwachsenenbildung im Rahmen des lebenslangen Lernens beschäftigen und denen eine Weiterentwicklung des Konzeptes des lebenslangen Lernens im Sinne eines der Aufklärung verpflichteten, offenen Projektes Bedürfnis und Anliegen ist. 
Open Access Dieser Artikel unterliegt den Bedingungen der Creative Commons Attribution License. Dadurch sind die Nutzung, Verteilung, und Reproduktion erlaubt, sofern der/die Originalautor/en und die Quelle angegeben sind. 